\title{
ON SELF-SIMILAR SOLUTIONS OF SEMILINEAR HEAT EQUATIONS AND SEPARATION STRUCTURE FOR RELATED ELLIPTIC EQUATIONS
}

\author{
Soohyun Bae
}

\begin{abstract}
We establish that if $n \geq 3$ and $p>1$ are large enough, then for each $\alpha>0$ the elliptic equation $\Delta u+\frac{1}{2} x \cdot \nabla u+\frac{m}{2} u+|x|^{l} u^{p}=0$ in $\mathbf{R}^{n}$ with $l>-2$ and $m=\frac{l+2}{p-1}$ possesses a positive radial solution $u_{\alpha}$ with $u_{\alpha}(0)=\alpha$ such that (i) $u_{\beta}>u_{\alpha}$ for $\beta>\alpha>0$; (ii) for every $\alpha>0, r^{m} u_{\alpha}(r) \rightarrow \ell$ as $r \rightarrow \infty$ for some $0<\ell=\ell(\alpha)<L$; (iii) $\ell(\alpha)$ is a one-to-one and onto increasing map from $(0, \infty)$ to $(0, L)$, where $L=[m(n-2-m)]^{1 /(p-1)}$.
\end{abstract}

\section{INTRODUCTION}

In this paper, we study the elliptic equation

$$
\Delta u+\frac{1}{2} x \cdot \nabla u+\frac{m}{2} u+|x|^{l} u^{p}=0,
$$

where $n \geq 3, \Delta=\sum_{i=1}^{n} \frac{\partial^{2}}{\partial x_{i}^{2}}$ is the Laplace operator, and $l>-2, m=\frac{l+2}{p-1}$, $p>1$. This equation is derived from the semilinear heat equation

$$
w_{t}=\Delta w+|y|^{l} w^{p} \quad \text { in } \quad \mathbf{R}^{n} \times(0, \infty) .
$$

By the scaling

$$
w_{\mu}(y, t)=\mu^{m} w\left(\mu y, \mu^{2} t\right)
$$

for $\mu>0$, a solution $w$ of (1.2) generates a one-parameter family of solutions. Of particular interest are self-similar solutions $w$ of (1.2), that is, $w_{\mu}(y, t)=w(y, t)$

Received January 15, 2003. Accepted April 15, 2003.

Communicated by S. B. Hsu.

2000 Mathematics Subject Classification: 35B40, 35J60, 35K15, 35K57.

Key words and phrases: Semilinear elliptic equations; Semilinear heat equations; Self-similar solutions; Separation; Singular solutions; Cauchy problems; Singular initial data; Uniqueness.

This work was supported by grant No. R01-2003-000-11731-0 from the Basic Research Program of the Korea Science and Engineering Foundation. 
for every $\mu>0$. Then a solution $w$ of (1.2) is self-similar if and only if $w$ is of the form

$$
w(y, t)=t^{-m / 2} u(y / \sqrt{t})
$$

with $u(x)$ satisfying (1.1). Self-similar solutions are useful in describing the large time behavior of global solutions of (1.2). For more information about (1.1), we refer the interested readers to $[8,13-16]$ and the references therein. To explain our motivations, we first consider positive steady states of (1.2) which satisfy

$$
\Delta u+|x|^{l} u^{p}=0 \quad \text { in } \quad \mathbf{R}^{n} .
$$

More generally, the elliptic equation for radial solutions has the form

$$
u_{r r}+\frac{n-1}{r} u_{r}+K(r) u^{p}=0
$$

where $u(x)=u(|x|), r=|x|$ and $K=K(r)$. It is known that equation (1.4) with $u(0)=\alpha>0$ has a unique positive solution $u_{\alpha} \in \mathbf{C}^{2}((0, \varepsilon)) \cap \mathbf{C}([0, \varepsilon))$ for small $\varepsilon>0$ under the following condition:

$$
(\mathrm{K})\left\{\begin{array}{l}
K(r) \text { is continuous on }(0, \infty), \\
K(r) \geq 0 \text { and } K(r) \not \equiv 0 \text { on }(0, \infty), \\
\int_{0} r K(r) d r<\infty
\end{array}\right.
$$

For $p>\frac{n+2+2 l}{n-2}$ with $l>-2$, if $r^{-l} K(r)$ is non-increasing on $(0, \infty)$, then (1.4) has the structure of Type $\mathbf{S}$ : (1.4) has a slowly decaying solution $u_{\alpha}(r)$ for every $\alpha>0$ which means that $u_{\alpha}(r)>0$ on $[0, \infty)$ and $r^{n-2} u_{\alpha}(r) \rightarrow \infty$ as $r \rightarrow \infty$. See [2]. In addition, if $r^{-l} K(r) \rightarrow 1$ as $r \rightarrow \infty$, then every positive solution $u$ of $(1.4)$ on $(0, \infty)$ satisfies

$$
\lim _{r \rightarrow \infty} r^{m} u(r)=L
$$

where $m=\frac{l+2}{p-1}$ and

$$
L=L(n, p, l)=[m(n-2-m)]^{\frac{1}{p-1}} .
$$

Recently, considerable attention has been given to separation of solutions of (1.4) (see [1, 3, 5-7]). In some cases, separated positive solutions are "stable" in a certain sense (see $[4,11,12])$. More precisely, the structure of Type $\mathrm{S}$ is divided into two types by the following exponent:

$$
p_{c}=p_{c}(n, l)=\left\{\begin{array}{cl}
\frac{(n-2)^{2}-2(l+2)(n+l)+2(l+2) \sqrt{(n+l)^{2}-(n-2)^{2}}}{(n-2)(n-10-4 l)} & \text { if } n>10+4 l, \\
\infty & \text { if } n \cdot 10+4 l
\end{array}\right.
$$


for some $l>-2$. The exponent $p_{c}(n, 0)$ has appeared in the study of elliptic equations in [9]. For $p \geq p_{c}(n, l)\left(>\frac{n+2+2 l}{n-2}\right)$, (1.4) has the structure of Type SS: (1.4) possesses a slowly decaying solution $u_{\alpha}$ for each $\alpha>0$ and any two of them do not intersect, as follows ([5; Theorem 1.2]):

Theorem A. Let $p \geq p_{c}(n, l)$ with $l>-2$. Assume that $K$ satisfies $(\mathrm{K})$, $r^{-l} K(r)$ is non-increasing in $r \in(0, \infty)$. Then the structure is of Type SS, and there is a singular solution $U(r)$ such that every positive solution $u_{\alpha}$ of (1.4) satisfies

$$
u_{\alpha}(r)<U(r) \cdot \frac{L(n, p, l)}{\left[r^{2} K(r)\right]^{\frac{1}{p-1}}}
$$

with the convention of $L / 0=\infty$. Moreover, $r^{m} u_{\alpha}(r)$ is strictly increasing as $r$ increases.

Our aim is to show the structure of Type SS for (1.1) when $p \geq p_{c}$. In other words, (1.1) as a lower order perturbation of (1.3) demonstrates separation structure similarly. Under (K), the initial value problem for positive radial solutions

$$
u_{r r}+\left(\frac{n-1}{r}+\frac{r}{2}\right) u_{r}+\frac{m}{2} u+K(r) u^{p}=0, \quad u(0)=\alpha>0,
$$

has a unique solution $u \in \mathbf{C}^{2}((0, \varepsilon)) \cap \mathbf{C}([0, \varepsilon))$ for $\varepsilon>0$ small. We denote the unique solution by $u_{\alpha}(r)$ also. The main result of this paper is the following

Theorem 1.1. Let $p \geq p_{c}(n, l)$ with $l>-2$. Assume that $K$ satisfies $(\mathrm{K})$, $r^{-l} K(r)$ is non-increasing in $r \in(0, \infty)$. Then, (1.7) has the structure of Type SS, and there is a singular solution $U(r)$ such that every positive solution $u_{\alpha}$ satisfies (1.6) with the convention of $L / 0=\infty$. Moreover, (i) $r^{m} u_{\alpha}(r)$ is strictly increasing as $r$ increases; (ii) for $\beta>\alpha>0, r^{m}\left(u_{\beta}(r)-u_{\alpha}(r)\right)$ does not converge to 0 at $\infty$.

As a result of Theorem 1.1, we show uniqueness of solutions for the Cauchy problem (1.2) with singular initial data

$$
w(y, 0)=c|y|^{-m} \quad \text { in } \quad \mathbf{R}^{n} \backslash\{0\}
$$

for $0<c<L(n, p, l)$. In [10], Galaktionov and Vazquez considered the quasi-linear heat equation

$$
w_{t}=\Delta w^{q}+w^{p} \quad \text { in } \quad \mathbf{R}^{n} \times(0, \infty)
$$

with singular initial data $w(y, 0)=c|y|^{-2 /(p-q)}$ for some $0<c<c_{s}$, where

$$
\left.c_{s}=\frac{\square q}{p-q}\left(n-2-\frac{2 q}{p-q}\right)\right]^{\frac{1}{p-q}} .
$$


They raised several questions on the Cauchy problem (1.9). For $q=1$, their conjecture is as follows [10; p. 41]:

(i) if $p \geq p_{c}(n, 0)$, then there exists a unique solution in the class $\{w$. $\left.L(n, p, 0)|y|^{-2 /(p-1)}\right\}$;

(ii) if $\frac{n+2}{n-2}<p<p_{c}(n, 0)$, then for $c=L(n, p, 0)$ there exist infinitely many solutions, and for $L(n, p, 0)-c>0$ small there exist an arbitrarily large finite number of solutions, while for $c>0$ small the solution is unique;

(iii) if $\frac{n}{n-2}<p \cdot \frac{n+2}{n-2}$, then there exist exactly two solutions.

In fact, they mentioned that (i) can be proved by Hardy's inequality as done in [17]. However, to apply Hardy's inequality to the question (i), one need to analyze the asymptotic behavior at $\infty$ of the difference of two solutions of the Cauchy problem. Our approach is to study directly separation structure for (1.1) rather than to employ the Hardy inequality. Then, the structure implies immediately the uniqueness.

Theorem 1.2. Let $p \geq p_{c}(n, l)$ with $l>-2$. The Cauchy problem (1.2) and (1.8) for $0<c<L(n, p, l)$ has a unique solution in the class $\left\{w \cdot L|y|^{-m}\right\}$.

\section{Preliminaries}

In this section, we consider basic properties on solutions of (1.7) under the assumption (K). In order to show the local existence of a mild solution which is defined by

$$
u(r)=\alpha-\int_{0}^{r}\left(t^{n-1} e^{t^{2} / 4}\right)^{-1} \int_{0}^{t}\left[\frac{m}{2} u+K u^{p}\right] s^{n-1} e^{s^{2} / 4} d s d t,
$$

one may define a continuous and compact operator on $\mathbf{C}([0, \varepsilon))$ with respect to the uniform convergence,

$$
T(u)(r)=\alpha-\int_{0}^{r}\left(t^{n-1} e^{t^{2} / 4}\right)^{-1} \int_{0}^{t}\left[\frac{m}{2} u+K u^{p}\right] s^{n-1} e^{s^{2} / 4} d s d t .
$$

By the contraction mapping principle, $T$ has a fixed point for small $\varepsilon>0$ which is a mild solution. On the other hand, any positive solution $u \in \mathbf{C}^{2}((0, \varepsilon)) \cap \mathbf{C}([0, \varepsilon))$ of (1.7) for small $\varepsilon>0$ satisfies (2.1) with $u(0)=\alpha$. Moreover,

$$
-r^{n-1} e^{r^{2} / 4} u_{r}(r)=\int_{0}^{r}\left[\frac{m}{2} u+K u^{p}\right] s^{n-1} e^{s^{2} / 4} d s
$$

and thus, 


$$
\left|r^{n-1} e^{r^{2} / 4} u_{r}(r)\right| \cdot r^{n-2} e^{r^{2} / 4}\left[\frac{m}{2} \alpha r^{2}+\alpha^{p} \int_{0}^{r} s K(s) d s\right]<\infty .
$$

Therefore, for each $\alpha>0,(1.7)$ has a local unique positive solution and the local solution is decreasing and extended locally as long as it remains positive. Then, it is natural to ask the maximal range on which the local solution is positive.

Proposition 2.1. Let $p>\frac{n+l}{n-2}$ with $l>-2$. Assume that $K$ satisfies $(\mathrm{K})$ and

$$
\lim _{r \rightarrow 0} r^{2} K(r)=0 .
$$

Then, every solution $u_{\alpha}$ of $(1.7)$ with $u_{\alpha}(0)=\alpha>0$ remains positive as long as the relation

$$
r^{2} K(r) u_{\alpha}^{p-1}(r)<L^{p-1}
$$

holds from $r=0$, where $L=L(n, p, l)$ is given by $(1.5)$.

Proof. Let $V(t):=r^{m} u_{\alpha}(r), t=\log r$. Then, $V$ satisfies

$$
V_{t t}+\left(a+\frac{1}{2} e^{2 t}\right) V_{t}-L^{p-1} V+k(t) V^{p}=0,
$$

where $a=n-2-2 m$ and $k(t):=e^{-l t} K\left(e^{t}\right)$. It follows from (2.4) that

$$
\lim _{t \rightarrow-\infty} k(t) V(t)^{p-1}=\lim _{r \rightarrow 0} r^{2} K(r) u_{\alpha}^{p-1}(r)=0
$$

and thus, $k V^{p-1}<L^{p-1}$ near $-\infty$. Suppose that there exists $T$ such that $V$ is positive and $k V^{p-1}<L^{p-1}$ on $(-\infty, T)$, but $V(T)=0$. Then, by (2.6), we have

$$
V_{t t}+\left(a+\frac{1}{2} e^{2 t}\right) V_{t}=\left(L^{p-1}-k(t) V^{p-1}\right) V>0 \quad \text { on } \quad(-\infty, T) .
$$

Multiplying (2.7) by $e^{f(t)}$, where

$$
f(t):=\int_{0}^{t}\left(a+\frac{1}{2} e^{2 s}\right) d s=a t+\frac{1}{4} e^{2 t}-\frac{1}{4},
$$

and integrating from $t$ to $T$, we obtain

$$
\begin{aligned}
e^{f(T)} V_{t}(T) & >e^{f(t)} V_{t}(t) \\
& =e^{\left(r^{2}-1\right) / 4} r^{a+m}\left[m u_{\alpha}(r)+r u_{\alpha}^{\prime}(r)\right]
\end{aligned}
$$


which goes to 0 as $r \rightarrow 0$ since from (2.3) $r u_{\alpha}^{\prime}(r) \rightarrow 0$ as $r \rightarrow 0$. Hence, we have $e^{a T} V_{t}(T)>0$, a contradiction.

From the proof of Proposition 2.1, we see the following

Corollary 2.2. On the maximal interval in which (2.5) holds, $r^{m} u_{\alpha}(r)$ is increasing as $r$ increases while $u_{\alpha}(r)$ is decreasing.

Another consequence of Proposition 2.1 is that separation happens for $\alpha>0$ sufficiently small.

Proposition 2.3. Let $p>\frac{n+l}{n-2}$ with $l>-2$. Assume that $K$ satisfies $(\mathrm{K})$ and (2.4). If $u_{\beta}$ is a positive entire solution of (1.7) satisfying

$$
r^{2} K(r) u_{\beta}^{p-1}(r) \cdot \frac{1}{p} L^{p-1},
$$

then for each $0<\alpha<\beta, u_{\alpha}$ is a positive entire solution of (1.7) and $r^{m}\left(u_{\beta}(r)-\right.$ $\left.u_{\alpha}(r)\right)$ is increasing in $r \in(0, \infty)$. Moreover, if $r^{-l} K(r) \rightarrow 1$ as $r \rightarrow \infty$, then for $0<\alpha<\beta$, $r^{m} u_{\alpha}(r) \rightarrow \ell$ as $r \rightarrow \infty$ for some $0<\ell(\alpha)<\ell(\beta) \cdot \frac{L}{p^{1 /(p-1)}}$.

Proof. For $\alpha>0$, let $V_{\alpha}(t):=r^{m} u_{\alpha}(r), t=\log r$. Set $W(t):=V_{\beta}(t)-V_{\alpha}(t)$ for given $\beta>\alpha>0$. By (2.6),

$$
W_{t t}+\left(a+\frac{1}{2} e^{2 t}\right) W_{t}-L^{p-1} W+k(t)\left(V_{\beta}^{p}-V_{\alpha}^{p}\right)=0 .
$$

Suppose that $W>0$ on $(-\infty, T)$ for some $T$ and $W(T)=0$. Then, from (2.10) and (2.11), we have

$$
W_{t t}+\left(a+\frac{1}{2} e^{2 t}\right) W_{t} \geq\left(L^{p-1}-k(t) p V_{\beta}^{p-1}\right) W \geq 0 .
$$

Multiplying (2.12) by $e^{f(t)}$ and integrating from $-\infty$ to $T$, we obtain $e^{f(T)} W_{t}(T)>$ 0 , a contradiction. Therefore, $W>0$ and thus the inequality $r^{2} K u_{\alpha}^{p-1} \cdot \frac{1}{p} L^{p-1}$ holds on any region. Then, by Corollary 2.2, $u_{\alpha}$ is an entire solution and $r^{m} u_{\alpha}$ is increasing. Moreover, $W$ is also increasing. If $r^{-l} K(r) \rightarrow 1$ as $r \rightarrow \infty$, then it follows from (2.5) and Corollary 2.2 that $r^{m} u_{\alpha}(r) \rightarrow \ell$ as $r \rightarrow \infty$ and $\ell(\alpha)<\ell(\beta)$.

\section{Structure of Type SS}

If (2.5) is true on $[0, \infty)$, then $u_{\alpha}$ is a positive entire solution of (1.7) and $r^{m} u_{\alpha}(r)$ is increasing as $r$ increases. The two conditions that $r^{-l} K(r)$ is nonincreasing and $p \geq p_{c}(n, l)$, guarantee that this relation is satisfied in the entire space and (1.7) has the structure of Type SS. 
Theorem 3.1. Let $p \geq p_{c}(n, l)$ with $l>-2$. Suppose that $K(r)$ satisfies $(\mathrm{K})$ and $r^{-l} K(r)$ is non-increasing. Then, for each $0<\alpha<\infty$, (1.7) possesses $a$ slowly decaying solution $u_{\alpha}$ with $u_{\alpha}(0)=\alpha$ such that $r^{m} u_{\alpha}(r)$ is strictly increasing and (2.5) holds on $[0, \infty)$, where $L=L(n, p, l)$.

Proof. Condition (2.4) follows immediately from (K) and

$$
\left.\int_{0}^{r} s K(s) d s \geq \int_{r / 2}^{r} s^{-l} K(s) s^{1+l} d s \geq \frac{1}{2+l} \quad 1-\frac{1}{2^{2+l}}\right] r^{2} K(r) .
$$

Let $\alpha>0$ and $V(t):=r^{m} u_{\alpha}(r), t=\log r$. Then, $V$ satisfies (2.6). Setting

$$
T=\sup \left\{\tau \mid k V^{p-1}<L^{p-1} \text { on }(-\infty, \tau)\right\},
$$

we see by Proposition 2.1 that $V$ is positive on $(-\infty, T)$. Suppose that $T<+\infty$ and $k(T) V(T)^{p-1}=L^{p-1}$. From Corollary $2.2, e^{a t} V_{t}$ is strictly increasing on $(-\infty, T)$ and $V_{t}(t)>0$ for $t$. T. Let $q(V)=V_{t}(t)$. Then, $q(V)>0$ on $\left(0,\left[\frac{1}{k(T)}\right]^{1 /(p-1)} L\right], q(V) \rightarrow 0$ as $V \rightarrow 0^{+}$, and

$$
\frac{d q}{d V}<-a+\frac{L^{p-1} V-k(t) V^{p}}{q} .
$$

Therefore, for every $\mu>0$, the line $q=\mu\left(\left[\frac{1}{k(T)}\right]^{1 /(p-1)} L-V\right)$ intersects the graph of $q(V)$ in the $q-V$ plane. Let $\left(V_{\mu}, q\left(V_{\mu}\right)\right)$ be the intersection with the smallest $V$-coordinate for each $\mu>0$. Then, we have $\frac{d q}{d V} \geq-\mu$ at $V_{\mu}$ and

$$
\frac{d q}{d V}\left(V_{\mu}\right)<-a+\frac{L^{p-1} V_{\mu}-k(t) V_{\mu}^{p}}{\mu\left(\left[\frac{1}{k(T)}\right]^{1 /(p-1)} L-V_{\mu}\right)} .
$$

Since $k(t)$ is non-increasing, e.g., $k(t) \geq k(T)$ for $t \cdot T$, we have

$$
\begin{aligned}
-\mu & <-a+\frac{k(T) V_{\mu}\left(\frac{1}{k(T)} L^{p-1}-V_{\mu}^{p-1}\right)}{\mu\left(\left[\frac{1}{k(T)}\right]^{1 /(p-1)} L-V_{\mu}\right)} \\
& =-a+\frac{(p-1) k(T) V_{\mu} \bar{V}_{\mu}^{p-2}}{\mu} \text { for some } \bar{V}_{\mu} \in\left(V_{\mu}, \frac{L}{[k(T)]^{1 /(p-1)}}\right) \\
& <-a+\frac{(p-1) L^{p-1}}{\mu}
\end{aligned}
$$

i.e., for all $\mu>0$,

$$
\mu^{2}-a \mu+(p-1) L^{p-1}>0 .
$$


It follows from (3.1) and $p \geq p_{c}>\frac{n+2+2 l}{n-2}$ that $a>0$ and the discriminant $a^{2}-4(p-1) L^{p-1}$ of the quadratic form in (3.1) is negative, which contradicts $p \geq p_{c}$. This shows that $k V^{p-1}<L^{p-1}$ on $(-\infty,+\infty)$ and (2.5) holds for $r>0$. Consequently, $e^{a t} V_{t}(t)>0$ for all $t \in \mathbf{R}$. Therefore, $r^{m} u_{\alpha}(r)$ is strictly increasing and $u_{\alpha}$ is a slowly decaying solution.

We are now ready to prove Theorem 1.1 and verify the structure of Type SS. Here, we make use of the argument of Lemma 4.1 in [7].

Proof of Theorem 1.1. It follows from Theorem 3.1 that for each $\alpha>0, u_{\alpha}$ is a slowly decaying solution. For $\alpha>0$, let $V_{\alpha}(t):=r^{m} u_{\alpha}(r), t=\log r$. Setting $W(t):=V_{\beta}(t)-V_{\alpha}(t)$ for $\beta>\alpha>0$ given, we see that $W$ is positive near $-\infty$ and satisfies

$$
W_{t t}+\Phi(t) W_{t}+(p-1) L^{p-1} W+G(t)=0
$$

where $\Phi(t):=a+\frac{1}{2} e^{2 t}$ and

$$
G(t):=-p L^{p-1} W(t)+e^{-l t} K\left(e^{t}\right)\left(V_{\beta}^{p}-V_{\alpha}^{p}\right) .
$$

Suppose that there exists $\bar{t} \in \mathbf{R}$ such that $W(t)>0$ on $(-\infty, \bar{t})$ and $W(\bar{t})=0$. It follows from (2.5) that for $t<\bar{t}$,

$$
\begin{aligned}
G(t) & <-p L^{p-1} W(t)+e^{-l t} K\left(e^{t}\right) W(t) p V_{\beta}^{p-1} \\
& =-p W(t)\left(L^{p-1}-e^{-l t} K\left(e^{t}\right)\left[r^{m} u_{\beta}(r)\right]^{p-1}\right) \\
& \cdot \quad 0 .
\end{aligned}
$$

For $t \cdot \bar{t}$, we have

$$
\left(W_{t}+\frac{1}{2} \Phi W\right)_{t}+\frac{1}{2} \Phi\left(W_{t}+\frac{1}{2} \Phi W\right) \geq 0
$$

since $a^{2}-4(p-1) L^{p-1} \geq 0$ from $p \geq p_{c}$. We may choose $-\infty<T<\bar{t}$ satisfying $W_{t}(T)=0$. Multiplying (3.3) by $e^{\frac{1}{2} \int_{T}^{t} \Phi}$ and integrating from $T$ to $t$ we obtain

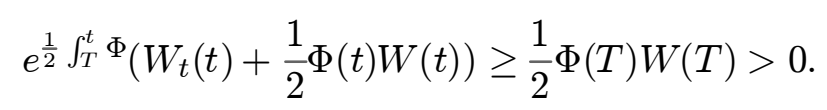

Therefore, from $W_{t}(\bar{t}) \cdot 0$ we deduce that for $t=\bar{t}$,

$$
\begin{aligned}
\frac{1}{2} \Phi(\bar{t}) W(\bar{t}) & \geq W_{t}(\bar{t})+\frac{1}{2} \Phi(\bar{t}) W(\bar{t}) \\
& \geq \frac{1}{2} \Phi(T) W(T) e^{-\frac{1}{2} \int_{T}^{\bar{t}} \Phi}>0,
\end{aligned}
$$


which implies $W(\bar{t})>0$, a contradiction. Therefore, we conclude that $V_{\beta}>V_{\alpha}$. Then, $u_{\beta}>u_{\alpha}>0$ in $\mathbf{R}^{n}$ for $\beta>\alpha>0$, and (1.7) has the structure of Type SS.

Setting $w(r):=W(t)$, we see that $w$ satisfies

$$
w_{r r}+\left(\frac{d}{r}+\frac{r}{2}\right) w_{r}=\theta \frac{w}{r^{2}},
$$

where $d=n-1-2 m$ and

$$
\begin{aligned}
\theta & =L^{p-1}-r^{-l} K(r) \frac{V_{\beta}^{p}-V_{\alpha}^{p}}{V_{\beta}-V_{\alpha}} \\
& =L^{p-1}-r^{-l} K(r) p \tilde{v}^{p-1}
\end{aligned}
$$

for some $V_{\alpha}<\tilde{v}<V_{\beta}$. From (2.5), $-(p-1) L^{p-1}<\theta<L^{p-1}$. Suppose that $w(r) \rightarrow 0$ as $r \rightarrow \infty$. We see that from (3.4),

$$
\begin{aligned}
-w(r) & =\int_{r}^{\infty}\left(t^{d} e^{t^{2} / 4}\right)^{-1} \int_{0}^{t} s^{d-2} e^{s^{2} / 4} \theta w d s d t \\
& =\int_{r}^{\infty}\left(t^{d} e^{t^{2} / 4}\right)^{-1} \int_{1}^{t} s^{d-2} e^{s^{2} / 4} o(1) d s d t+o\left(e^{-r^{2} / 4}\right) \text { at } r=\infty .
\end{aligned}
$$

By L'Hôpital's rule, we have

$$
\begin{aligned}
& \lim _{r \rightarrow \infty} r^{2} \int_{r}^{\infty} t^{-d} e^{-t^{2} / 4} \int_{1}^{t} s^{d-2} e^{s^{2} / 4} d s d t \\
& =\lim _{r \rightarrow \infty} \frac{-r^{-d} e^{-r^{2} / 4} \int_{1}^{r} s^{d-2} e^{s^{2} / 4} d s}{-2 r^{-3}} \\
& =\lim _{r \rightarrow \infty} \frac{r^{d-2} e^{r^{2} / 4}}{2(d-3) r^{d-4} e^{r^{2} / 4}+r^{d-2} e^{r^{2} / 4}} \\
& =1 .
\end{aligned}
$$

Hence, we have $w(r)=o\left(1 / r^{2}\right)$ at $r=\infty$ and by repeating the above argument with this finer estimate, $w(r)=o\left(1 / r^{4}\right)$. Iterating the process, we derive that $w(r)=o\left(1 / r^{\tau}\right)$ at $r=\infty$ for any $\tau>0$. Since by (3.5),

$$
w_{r}(r)=r^{-d} e^{-r^{2} / 4} \int_{0}^{r} s^{d-2} e^{s^{2} / 4} \theta w d s
$$

we have also $w_{r}(r)=o\left(1 / r^{\tau}\right)$ at $r=\infty$ for any $\tau>0$. To apply a comparison argument, consider the function $\Psi(r):=r^{b-(n-2) / 2} e^{-r^{2} / 4}$ satisfying

$$
\Delta \Psi+\frac{1}{2} x \cdot \nabla \Psi+\left(\frac{\lambda}{|x|^{2}}+\frac{n+2+2 b}{4}\right) \Psi=0,
$$


where $\lambda=m p(n-2-m)$ and $b=\sqrt{(n-2)^{2} / 4-\lambda}$. Because $p \geq p_{c}$ and $a^{2}-4(p-1) L^{p-1} \geq 0$, we have $(n-2)^{2} / 4-\lambda \geq 0$. Setting $q(t):=r^{m} \Psi(r)=$ $r^{b-\frac{a}{2}} e^{-r^{2} / 4}$, we see that $q$ is a positive solution of the equation

$$
q_{t t}+\left(a+\frac{1}{2} e^{2 t}\right) q_{t}+(p-1) L^{p-1} q=\left(\frac{m}{2}-\frac{n+2+2 b}{4}\right) e^{2 t} q<0 .
$$

Observe that $q_{t}=\left(b-\frac{a}{2}\right) q-\frac{1}{2} e^{2 t} q$. Multiplying (3.2) by $q$, (3.6) by $W$, and taking the difference, we have

$$
\left(W_{t} q-W q_{t}\right)_{t}+\left(a+\frac{1}{2} e^{2 t}\right)\left(W_{t} q-W q_{t}\right)+q G(t)>0 .
$$

From (2.9), $W(t), W_{t}(t)=O\left(e^{m t}\right)$ at $t=-\infty$. Obviously,

$$
e^{f(t)} q(t), e^{f(t)} q_{t}(t)=O\left(e^{\left(b+\frac{a}{2}\right) t}\right) \quad \text { at } \quad t=-\infty
$$

and

$$
e^{f(t)} q(t)=O\left(e^{\left(b+\frac{a}{2}\right) t}\right), e^{f(t)} q_{t}(t)=O\left(e^{\left(b+\frac{a}{2}+2\right) t}\right) \quad \text { at } \quad t=+\infty,
$$

where $f(t)$ is defined in (2.8). Multiplying (3.7) by $e^{f(t)}$ and integrating over $(T, t)$, we obtain

$$
\left.e^{f}\left(W_{t} q-W q_{t}\right)\right|_{T} ^{t}>-\int_{T}^{t} e^{f(s)} q(s) G(s) d s .
$$

Letting $T \rightarrow-\infty$ and then $t \rightarrow+\infty$, we have from the above decay estimates that

$$
0>-\int_{-\infty}^{+\infty} e^{f(s)} q(s) G(s) d s>0
$$

which is impossible. Therefore, $W(t)$ does not converge to 0 at $t=+\infty$.

Since any solution $u_{\alpha}$ has uniform bounds by (2.5) on any compact set in $(0, \infty)$, the existence of a singular solution of (1.6) is verified by a standard method. Combining (2.2), (2.5) and the fact that $r^{-l} K$ is non-increasing, we have

$$
\begin{aligned}
-r^{n-1} e^{r^{2} / 4} u_{r}(r)= & \int_{0}^{r}\left[\frac{m}{2} u+K u^{p}\right] s^{n-1} e^{s^{2} / 4} d s \\
& \cdot \int_{0}^{r}\left[\frac{m L}{2} s^{-\frac{2}{p-1}}+L^{p} s^{-\frac{2 p}{p-1}}\right] s^{n-1} e^{s^{2} / 4} K(s)^{\frac{-1}{p-1}} d s \\
& \cdot r^{\frac{l}{p-1}} K(r)^{\frac{-1}{p-1}}\left[\frac{m L}{2} \int_{0}^{r} s^{n-1-m} e^{s^{2} / 4} d s\right. \\
& \left.+L^{p} \int_{0}^{r} s^{n-1-\frac{2 p+l}{p-1}} e^{s^{2} / 4} d s\right] .
\end{aligned}
$$

Note first that $K$ is positive near 0 , and if $K(R)=0$ for some $R>0$, then $K(r)=0$ on $[R, \infty)$, and second that $u_{\alpha}(r)$ is decreasing in $r$. Hence, $u_{\alpha}^{\prime}$ is 
uniformly bounded on any compact subset of $(0, \infty)$ in $\alpha$ and consequently, $\left\{u_{\alpha}\right\}$ is equicontinuous on any compact subset. Since $u_{\alpha}$ is monotonically increasing, the Arzelà-Ascoli Theorem implies that $U(r):=\lim _{\alpha \rightarrow \infty} u_{\alpha}(r)$ is well-defined and continuous on $(0, \infty)$ and for each $\alpha>0$,

$$
u_{\alpha}(r)<U(r) \cdot \frac{L(n, p, l)}{\left[r^{2} K(r)\right]^{\frac{1}{p-1}}} .
$$

By (1.7), $u_{\alpha}^{\prime \prime}$ is uniformly bounded on any compact subset of $(0, \infty)$ in $\alpha$ and $\left\{u_{\alpha}^{\prime}\right\}$ is equicontinuous on any compact subset. It follows again from the Arzelà-Ascoli Theorem that for a subsequence $\left\{\alpha_{j}\right\}$ going to $\infty, u_{\alpha_{j}}^{\prime}$ converges uniformly on any compact subset and thus $U$ is differentiable on $(0, \infty), u_{\alpha_{j}}^{\prime} \rightarrow U^{\prime}$ uniformly on any compact subset. Since by (1.7) $u_{\alpha_{j}}^{\prime \prime}$ converges also uniformly, $U^{\prime}$ is differentiable on $(0, \infty)$ and $u_{\alpha_{j}}^{\prime \prime} \rightarrow U^{\prime \prime}$ uniformly on any compact subset of $(0, \infty)$. Then, $U$ satisfies

$$
U^{\prime \prime}+\left(\frac{n-1}{r}+\frac{r}{2}\right) U^{\prime}+\frac{m}{2} U+K(r) U^{p}=0 \quad \text { on } \quad(0, \infty)
$$

and $U$ is a singular solution of (1.7). Now, the proof of Theorem 1.1 is complete.

In case $r^{-l} K(r) \rightarrow 1$ as $r \rightarrow \infty$ for some $l>-2$, we analyze further separation phenomena.

Theorem 3.2. Assume the hypotheses of Theorem 1.1 and $r^{-l} K(r) \rightarrow 1$ as $r \rightarrow \infty$. Then, there exists $0<\ell^{*} . L(n, p, l)$ such that $r^{m} U(r) \rightarrow \ell^{*}$ as $r \rightarrow \infty$ and for every $\alpha>0, r^{m} u_{\alpha}(r) \rightarrow \ell$ for some $0<\ell=\ell(\alpha)<\ell^{*}$. Moreover, $\ell(\beta)>\ell(\alpha)$ for $\beta>\alpha>0$.

Proof. By (2.5), we see that $r^{m} u_{\alpha}(r) \rightarrow \ell \cdot L$ since $r^{m} u_{\alpha}(r)$ is increasing in $r$. This fact combined with (3.8) implies that $r^{m} U(r)$ is increasing and converges to $\ell^{*}$ for some $0<\ell^{*} . L$. For $\alpha>0$, let $V_{\alpha}(t):=r^{m} u_{\alpha}(r), t=\log r$. Set $W(t)$ : $=V_{\beta}(t)-V_{\alpha}(t)$ for given $\beta>\alpha>0$. From Theorem 1.1, $W$ is strictly increasing and thus $\ell(\beta)>\ell(\alpha)$. Therefore, $0<\ell(\alpha)<\ell(\beta)<\ell^{*}$ for $0<\alpha<\beta$.

In case $K(x)=|x|^{l}$, uniqueness for the Cauchy problem (1.2) and (1.8) follows directly from Theorem 3.2. The Cauchy problem has a minimal solution $w_{1}$ and a maximal solution $w_{2}$ in the class $\left\{w \cdot L|y|^{-m}\right\}$ (see $[10,15]$ ). Moreover, $\ell(\alpha)$ is a one-to-one and onto increasing map from $(0, \infty)$ to $(0, L)$. We observe that $w_{1}, w_{2}$ are self-similar and

$$
w_{i}(y, t)=t^{-m / 2} u_{i}(y / \sqrt{t}), \quad i=1,2,
$$


where $u_{i}$ are positive entire radially symmetric solutions of (1.1). Since $r^{m} u_{i}(r) \rightarrow$ $c$ as $r \rightarrow \infty$, it follows from Theorem 3.2 (the uniqueness of solutions with the asymptotic behavior) that $u_{2}=u_{1}$ and thus $w_{2}=w_{1}$.

\section{REFERENCES}

1. S. Bae, Infinite multiplicity and separation structure of positive solutions for a semilinear elliptic equation in $\mathbf{R}^{n}$, J. Differential Equations, to appear.

2. S. Bae, On the elliptic equation $\Delta u+K u^{p}=0$ in $\mathbf{R}^{n}$, preprint.

3. S. Bae, Separation structure of positive radial solutions of a semilinear elliptic equation in $\mathbf{R}^{n}$, J. Differential Equations, 194 (2003), 460-499.

4. S. Bae, Weighted $L^{\infty}$ stability of positive steady states of a semilinear heat equation in $\mathbf{R}^{n}$, preprint.

5. S. Bae and T. K. Chang, On a class of semilinear elliptic equations in $\mathbf{R}^{n}, J$. Differential Equations 185 (2002), 225-250.

6. S. Bae, T. K. Chang and D. H. Pahk, Infinite multiplicity of positive entire solutions for a semilinear elliptic equation, J. Differential Equations 181 (2002), 367-387.

7. S. Bae and W.-M. Ni, Existence and infinite multiplicity for an inhomogeneous semilinear elliptic equation on $\mathbf{R}^{n}$, Math. Ann. 320 (2001), 191-210.

8. M. Escobedo and O. Kavian, Variational problems related to self-similar solutions of the heat equation, Nonlinear Analysis TMA 11 (1987), 1103-1133.

9. D. D. Joseph and T. S. Lundgren, Quasilinear Dirichlet problems driven by positive sources, Arch. Rational Mech. Anal. 49 (1973), 241-269.

10. V. A. Galaktionov and J. L. Vazquez, Continuation of blowup solutions of nonlinear heat equations in several space dimensions, Comm. Pure Appl. Math. 50 (1997), $1-67$.

11. C. Gui, W.-M. Ni and X. Wang, On the stability and instability of positive steady states of a semilinear heat equation in $\mathbf{R}^{n}$, Comm. Pure Appl. Math. 45 (1992), 1153-1181.

12. C. Gui, W.-M. Ni and X. Wang, Further study on a nonlinear heat equation, $J$. Differential Equations 169 (2001), 588-613.

13. A. Haraux and F. B. Weissler, Non-uniqueness for a semilinear initial value problem, Indiana Univ. Math. J. 31 (1982), 167-189.

14. O. Kavian, Remarks on the large time behavior of a nonlinear diffusion equations, Ann. Inst. Henri Poincaré - Analyse non linéaire 4 (1987), 423-452.

15. Y. Naito and T. Suzuki, Radial symmetry of self-similar solutions for semilinear heat equations, J. Differential Equations 163 (2000), 407-428. 
16. L. A. Peletier, D. Terman and F. B. Weissler, On the equation $\Delta u+\frac{1}{2} x \cdot \nabla u+f(u)=0$, Arch. Rational Mech. Anal. 94 (1986), 83-99.

17. I. Peral and J. L. Vazquez, On the stability and instability of the singular solution of the semilinear heat equation with exponential reaction term, Arch. Rational Mech. Anal. 129 (1995), 201-224.

\author{
Soohyun Bae \\ Department of Mathematics, \\ Yonsei University, \\ Seoul 120-749, \\ Republic of Korea \\ Current Address: \\ School of Liberal Arts, \\ Hanbat National University, \\ Daejeon 305-719, \\ Republic of Korea
}

